CLINICAL STUDY

\title{
Sympatho-vagal control of heart rate variability in patients treated with suppressive doses of $L$-thyroxine for thyroid cancer
}

\author{
Massimo Casu, Caterina Cappi, Vittoriana Patrone, Eva Repetto, Massimo Giusti, Francesco Minuto and \\ Giovanni Murialdo \\ Department of Endocrinological and Metabolic Sciences, University of Genova, Viale Benedetto XV, 6, I-16132, Genova, Italy \\ (Correspondence should be addressed to G Murialdo; Email: gmurialdo@unige.it)
}

\begin{abstract}
Objective: This study aimed to analyze the autonomic control of heart rate variability (HRV) in subjects receiving chronic L-thyroxine (L-T4) treatment after total thyroidectomy and ${ }^{131}$ I therapy for differentiated thyroid carcinoma.

Methods: Blood pressure (BP) and sympatho-vagal activity (evaluated by power spectral analysis (PSA) of time-domain parameters of HRV) were studied in clinostatism and after orthostatism in 24 healthy controls, and in 12 patients taking L-T4 $(125-200 \mu \mathrm{g} /$ day) to maintain serum TSH levels at $<0.01 \mu \mathrm{IU} / \mathrm{ml}$. The study of HRV by PSA is a non-invasive method of analyzing sympatho-vagal control of HRV by quantifying high-frequency (HF) $(0.15-0.4 \mathrm{~Hz})$ and low-frequency (LF) $(0.04-0.15 \mathrm{~Hz})$ powers.

Results: Patients on L-T4 treatment had undetectable TSH levels, serum free T4 (fT4) above the normal range or at the upper limit in one case, and normal free tri-iodothyronine (fT3) levels. Heart rate and $\mathrm{R}-\mathrm{R}$ intervals were not different in the two groups, both in clinostatism and in ortostatism. Systolic and mean BP were higher in patients than in controls and were inversely correlated with actual serum fT4 levels. During clinostatism, thyroid patients showed significantly lower LF power $(P=0.035), \mathrm{LF} /(\mathrm{LF}+\mathrm{HF})(P=0.008)$ and $\mathrm{LF} / \mathrm{HF}(P=0.01)$ than controls. When patients moved from lying to standing, there was a significantly different decrease in orthostatic LF power $(P=0.001), \mathrm{LF} /(\mathrm{LF}+\mathrm{HF})(P=0.044)$ and $\mathrm{LF} / \mathrm{HF}(P=0.047)$ versus controls.

Conclusions: Changes in autonomic control of HRV, characterized by decreased sympathetic activity and impaired sympatho-vagal balance with preserved vagal tone, are detectable in patients with hyperthyroxinemia due to suppressive L-T4 therapy and increased systolic and mean, but not diastolic, BP.
\end{abstract}

European Journal of Endocrinology 152 819-824

\section{Introduction}

Thyroid hormones affect cardiac function through their direct effects on the heart or by influencing sympathovagal tone, with hyperthyroid patients presenting signs and symptoms mimicking a sympathetic overactivity $(1-3)$.

Overt hyperthyroidism is known to alter both heart performance and morphology (1-7). However, myocardial changes and dysfunction (faster heart rate, increased left ventricular mass and enhanced function, altered diastolic filling), more frequent occurrence of atrial fibrillation and impaired cardiac autonomic control have also been reported in patients with subclinical hyperthyroidism $(7-11)$. This condition is characterized by lowered or undetectable thyroid-stimulating hormone (TSH) levels and free thyroid hormone concentrations persistently within the normal range (12). Cardiovascular alterations, although less intensive, have also been detected when doses of L-thyroxine (L-T4) inhibiting TSH secretion were administered $(6,7,13-18)$.

Despite clinical evidence for a sympathetic overactivity, a great deal of uncertainty persists as to the interaction between the autonomic system and thyroid hormones in the control of heart rate and function. The study by power spectral analysis (PSA) of electrocardiographic (ECG) R-R intervals, and of acute physiological adaptative response to postural changes, is a reliable, sensitive and non-invasive tool for measuring sympatho-vagal balance in humans $(19,20)$.

Changes in autonomic control of heart rate variability (HRV) have been demonstrated by PSA in overt hyperthyroidism and to a lesser extent in patients with spontaneous subclinical hyperthyroidism, but some discrepancies exist among the various reports 
due to clinical differences in patients studied and in methodological approaches $(21-26)$.

In this study, we analyzed HRV by PSA in a group of subjects chronically treated with L-T4 to inhibit TSH secretion after thyroid ablation by surgery followed by radioactive ${ }^{131} \mathrm{I}$ (RAI) therapy for differentiated thyroid carcinoma (DTC). Suppression of TSH secretion by exogenous L-T4 administration involves the use of hormone dosages that induce a supra-physiological increase in circulating levels of free $\mathrm{T} 4$ (fT4), converted to tri-iodithyronine (T3) by peripheral deiodinases. Thus, this condition appears to be different both from overt hyperthyroidism and from spontaneous subclinical hyperthyroidism, in which thyroid hormone levels in serum are normal by definition.

\section{Subjects and methods}

\section{Subjects}

Twelve patients ( 2 male, 10 female), aged between 36 and 69 years (mean age \pm s.D., 56.8 \pm 9.0 years), were enrolled in this study. They underwent total thyroidectomy followed by RAI for DTC of papillary or follicular type and were treated with L-T4 attaining suppression of TSH levels between 4 months and 3 years before the study. They attended routine follow-up for DTC and gave informed and written consent to the PSA study according to the Helsinki declaration.

Patients were treated with L-T4 at doses ranging between 125 and $200 \mu \mathrm{g} /$ day orally $(1.5-2.2 \mu \mathrm{g} / \mathrm{kg}$ body weight) and presented persistently suppressed TSH levels $(<0.005 \mu \mathrm{IU} / \mathrm{ml})$. Patients took their current doses of $\mathrm{L}-\mathrm{T} 4$ at $0700 \mathrm{~h}$ and $\mathrm{TSH}$, free fractions of thyroid hormones and thyroglobulin $(\mathrm{Tg})$ serum levels were assayed between 0800 and $0900 \mathrm{~h}$ on the same days before the autonomic tests. All patients were free from disease recurrence and their Tg levels were below the lowest normal range limit $(<0.5 \mathrm{pg} / \mathrm{ml})$.

Cardiovascular data in patients were compared with those of 24 sex- (4 male, 20 female) and age-matched healthy controls, 38-67 years old (mean age \pm s.D., $54.3 \pm 15.6$ years), with normal thyroid and heart function. Patients and controls did not take drugs interfering with cardiovascular function. No smoking or consumption of coffee, tea or other stimulating substances were allowed during the $6 \mathrm{~h}$ before the study.

\section{Assay methods}

Serum levels of TSH, fT4 and fT3 were measured using ultrasensitive chemiluminescence immunoassays (Roche). The third-generation ultrasensitive TSH method was standardized against the 2nd IRP WHO Reference Standard 80/558; it had a lower detection limit of $0.005 \mu \mathrm{IU} / \mathrm{ml}$ and sensitivity of $0.01 \mu \mathrm{IU} / \mathrm{ml}$.
A one-step immunoradiometric method (Techno Genetics, Milan, Italy) was used for Tg assay.

\section{HRV analysis}

An ECG recording of $\mathrm{R}-\mathrm{R}$ intervals was made at $1000 \mathrm{~h}$ in a quiet dedicated room, $1 \mathrm{~h}$ at least after venipuncture for routine hormonal evaluation. Subjects were placed supine on the tilting table and instructed to relax, stay awake, breathe regularly, and not to speak. After supine resting for about $10 \mathrm{~min}$ to stabilize blood pressure and heart rate (HR), clinostatic $\mathrm{R}-\mathrm{R}$ intervals were acquired. The recording was repeated immediately thereafter with the move from lying to standing achieved by a 30-s passive shift using a mechanically driven tilt table.

For both clinostatic and orthostatic posture, ECG was continuously recorded for $330 \mathrm{~s}$ by means of an ECG connected to a personal computer equipped with software that sampled the analogical signal at about $200 \mathrm{~Hz}$ using an analog-digital converter. Every R-R interval was measured in milliseconds and memorized as a tachogram $(\mathrm{R}-\mathrm{R}$ interval duration vs number of progressive cardiac beats). Two series of data corresponding to clinostatic (tachogram A) and orthostatic (tachogram B) R-R intervals were analyzed in all subjects by a parametric method, based on the autoregressive model for the quantification of HRV signal (27).

The main power densities in the high-frequency (HF) $(0.15-0.4 \mathrm{~Hz})$ and low-frequency (LF) $(0.04-0.15 \mathrm{~Hz})$ bands were identified for each density spectrum. HF power is thought to be a marker of the modulation of vagal tone, while LF power is mainly a measure of the modulation of vagal and sympathetic tone by baroreflex activity $(28,29)$. In addition, both $\mathrm{LF} /(\mathrm{LF}+\mathrm{HF})$ and LF/HF ratios were calculated. The first ratio is an index of sympathetic power with respect to the sum of LF and HF absolute values, while LF/HF is to be considered as an index of sympatho-vagal balance in the frequency domain (28).

Mean $\mathrm{R}-\mathrm{R}$ intervals (ms) and corresponding $\mathrm{HR}$ (beats/min) were calculated on the 330s tachogram series obtained in clinostatism and orthostatism.

Blood pressure (BP) was preliminarily measured in both arms by an automatic pressurometer (AMI System 7100) to exclude inter-arm differences. Then, mean values of three consecutive systolic, mean and diastolic BP (SBP, MBP and DBP respectively) values in clinostatism were calculated; $\mathrm{BP}$ was again measured after $1 \mathrm{~min}$ of orthostatism.

\section{Statistical analysis}

All results are given as the mean values \pm S.D. Data were analyzed using the Prism 4.0 software (GraphPad, San Diego, CA, USA) and preliminarily processed by the Kolmogorov-Smirnov test to assess their normality and skewness. Because of the non-parametric distribution of 
some data and indices of autonomic function, the Mann-Whitney $U$ test for unpaired data was applied for comparisons of data between patients and healthy controls. Statistical differences in PSA data observed in clinostatism and orthostatism in each of the two groups were analyzed by the Wilcoxon test. Correlations between thyroid hormone levels and BP, HR and PSA data were analyzed by Pearson' $r$ correlation test. The level of significance was set at $P<0.05$.

\section{Results}

\section{Endocrine data}

Serum TSH levels were undetectable $(<0.005 \mu \mathrm{IU} / \mathrm{ml})$ in all patients (normal range, $0.3-4.2 \mu \mathrm{IU} / \mathrm{ml}$ ). Serum fT4 levels were between 21.9 and $28.8 \mathrm{pmol} / \mathrm{l}$ and exceeded the normal range in all but one patient (mean levels \pm s.D., $24.5 \pm 2.3 \mathrm{pmol} / \mathrm{l}$; normal range, $12.0-22.0 \mathrm{pmol} / \mathrm{l})$. The fT3 levels were normal in all patients, ranging between 3.5 and $6.1 \mathrm{pmol} / \mathrm{l}$ (mean \pm S.D., $5.1 \pm 0.7 \mathrm{pmol} / \mathrm{l}$; normal range, 3.5-6.8 $\mathrm{pmol} / \mathrm{l})$.

\section{BP data and PSA analysis of HRV}

SBP and MBP, but not DBP, were slightly higher in DTC patients than in healthy controls (Table 1).

No significant difference was observed between $R-R$ interval duration and HR, neither in patients nor in controls, during clinostatism and orthostatism (Table 2). Moving from lying to standing significantly shortened $\mathrm{R}-\mathrm{R}$ intervals and accelerated HR in controls. A similar effect on HR was obtained in thyroid patients, who showed changes in $\mathrm{R}-\mathrm{R}$ intervals after orthostatism that were at the limit of statistical significance (Table 2).

PSA data of HRV during clinostatism and orthostatism in the two groups are reported in Figs 1 and 2. Lying DTC patients presented significantly lowered LF power, but no difference was observed in HF power between the two groups (Fig. 1). Moreover, DTC patients had significantly lowered $\mathrm{LF} /(\mathrm{LF}+\mathrm{HF})$ and $\mathrm{LF} / \mathrm{HF}$ ratios indicating an alteration of sympathetic control of HRT during clinostatism (Fig. 2).

Moving from lying to standing did not modify LF power in patients, while a physiological (but not significant) increase was observed in controls. Postural change induced a significant fall in HF power in both groups of subjects, along with an increase in $\mathrm{LF} /(\mathrm{LF}+\mathrm{HF})$ and $\mathrm{LF} / \mathrm{HF}$ ratios when compared with clinostatic data (Figs 1 and 2).

Changing from lying to standing amplified the difference existing between LF powers in patients and in controls, orthostatic $\mathrm{LF} /(\mathrm{LF}+\mathrm{HF})$ and $\mathrm{LF} / \mathrm{HF}$ remaining significantly lowered in the first group. In addition, no statistical difference was observed in this instance between orthostatic HF values in patients and controls.

Clinostatic SBP $(r=-0.6200 ; P=0.0315)$ and MBP $(r=-0.6316 ; P=0.0276)$ values were inversely correlated with serum fT4 levels in DTC patients. No other significant correlations were observed between cardiovascular parameters and thyroid hormone levels, L-T4 dosages or L-T4 treatment duration.

\section{Discussion}

In patients treated with TSH-suppressive doses of L-T4 for DTC, we did not find significant differences in $\mathrm{R}-\mathrm{R}$ intervals and HR during clinostatism and after moving from lying to standing, in comparison with healthy controls. This finding agrees with previous observations on $\mathrm{R}-\mathrm{R}$ intervals in resting subjects with spontaneous subclinical hyperthyroidism (25). Increased HR (9) and reduced average R-R intervals (24) were instead found by an ECG 24-h Holter recording in free-moving subclinical hyperthyroid subjects. In both groups we studied, HR was enhanced by standing up, but rapid posture changes increased HR in controls more than in patients. This behaviour was consistent with an impaired sympathetic activity expressed by lowered LF but unchanged HF power emerging from PSA of HRV in patients.

LF power is an index of both sympathetic and vagal modulation by baroreceptor activity, mainly reflecting the effects of the former (29); HF power is a marker of vagal activity modulation by the frequency and depth of ventilation $(30,31)$.

The impairment of LF baroreflex-mediated autonomic activity was emphasized by lowered LF/(LF/HF) and $\mathrm{LF} / \mathrm{HF}$ ratios. These indices are expressive of the balance of autonomic control on HRV and better define shifts from vagal to sympathetic dominance,

Table 1 SBP, MBP and DBP values in clinostatism and 1 min after orthostatism in 12 patients with DTC on chronic L-T4 treatment, and in 24 healthy controls. Statistical analysis: Mann-Whitney $U$ test for unpaired data.

\begin{tabular}{|c|c|c|c|c|c|c|}
\hline & \multicolumn{3}{|c|}{ Clinostatism } & \multicolumn{3}{|c|}{ Orthostatism } \\
\hline & SBP & MBP & DBP & SBP & MBP & DBP \\
\hline $\begin{array}{l}\text { DTC patients } \\
\text { Healthy controls } \\
P\end{array}$ & $\begin{array}{c}135.5 \pm 11.6 \\
125.9 \pm 9.4 \\
0.011\end{array}$ & $\begin{array}{c}114.5 \pm 9.5 \\
108.1 \pm 7.3 \\
0.033\end{array}$ & $\begin{array}{c}72.6 \pm 8.1 \\
72.7 \pm 8.6 \\
0.906\end{array}$ & $\begin{array}{c}137.0 \pm 12.0 \\
123.6 \pm 7.6 \\
0.015\end{array}$ & $\begin{array}{c}118.2 \pm 10.1 \\
108.1 \pm 6.7 \\
0.014\end{array}$ & $\begin{array}{c}80.6 \pm 9.3 \\
77.0 \pm 9.0 \\
0.289\end{array}$ \\
\hline
\end{tabular}


Table $2 \mathrm{R}-\mathrm{R}$ interval duration and heart rate (HR) during clinostatism and orthostatism in 12 patients with DTC treated with suppressive doses of L-T4 and in 24 healthy controls. Statistical analysis: Mann-Whitney $U$ test for unpaired data and Wilcoxon test for paired data.

\begin{tabular}{|c|c|c|c|c|c|c|}
\hline & \multicolumn{3}{|c|}{$\mathbf{R}-\mathbf{R}$ interval (ms) } & \multicolumn{3}{|c|}{ HR (beats/min) } \\
\hline & Clinostatism & Orthostatism & $P$ & Clinostatism & Orthostatism & $P$ \\
\hline DTC patients & $892.4 \pm 135.3$ & $840.7 \pm 229.3$ & 0.064 & $68.7 \pm 10.8$ & $75.2 \pm 15.7$ & 0.034 \\
\hline Healthy controls & $837.8 \pm 95.8$ & $756.0 \pm 114.8$ & 0.0002 & $72.4 \pm 7.6$ & $81.1 \pm 12.1$ & 0.0002 \\
\hline$P$ & 0.347 & 0.411 & & 0.347 & 0.411 & \\
\hline
\end{tabular}

their lowering suggesting a sympathetic withdrawal $(19,28)$.

Cacciatori et al. (21) and Burggraaf et al. (23) have found an impairment of HF parasympathetic activity in hyperthyroid patients, with some differences concerning the decrease in LF power which was unchanged or only slightly reduced.

After an ECG 24-h Holter recording, Petretta et al. (24) reported a lowered LF power and a not significant decrease in LF/HF in patients with endogenous sub-clinical hyperthyroidism, but a cardiac parasympathetic withdrawal with an increase of LH/HF was present in those with overt hyperthyroidism (24). The finding has recently been confirmed in a very large number of hyperthyroid patients before treatment (26).

Goichot et al. (25) reported that, during a 5-min observation, there were no significant differences in $\mathrm{LF}$ power and $\mathrm{LF} /(\mathrm{LF}+\mathrm{HF})$ ratios between healthy controls and patients with either overt or subclinical hyperthyroidism. In this series, only HF was significantly decreased in overt hyperthyroidism, but not in subclinical hyperthyroidism.

These data indicate that a decrease in vagal activity rather than an activation of the sympathetic tone characterizes the autonomic control of heart function in overt hyperthyroidism (21-26). Thus, reduced cardiac sympathetic activity at the early stage of hyperthyroidism or hyperthyroxinemia, as well as during subclinical hyperthyroidism, apparently differs from the vagal impairment and clinically evident sympathetic overactivity in the overt stages of the disease.

An inverse correlation was found between fT4 levels and SBP and MBP in our patients. A negative correlation between thyroid hormone concentrations in serum and sympathetic nerve activity in muscle was also reported by Matsukawa et al. (32). Moreover, motor vagal neuron excitability in response to carotideal baroreceptor activation was found to be impaired in hyperthyroidism (33) and resting HR appeared mainly affected by a marked reduction in the efferent activity of the parasympathetic component $(33,34)$.

Moving from lying to standing involves a complex adaptative response to maintain $\mathrm{BP}$ and brain perfusion. This physiological effect mainly consists of a sympathetic activation, which increases HR and output, combined with an inhibition of the vagal tone and increased LF/HF $(28,35)$.

After postural variation in patients, we observed an inadequate response of LF power, which even decreased with respect to the values recorded in clinostatism $(-9.1 \%$ in patients vs $+19.6 \%$ in controls). A more evident fall was observed in both groups in the HF power $(-66.5 \%$ in patients vs $-42.3 \%$ in controls).

Our patients had SBP and MBP slightly but significantly higher than those in healthy controls, while diastolic BP was similar in the two groups. The chronic inhibition of TSH secretion by increased thyroid
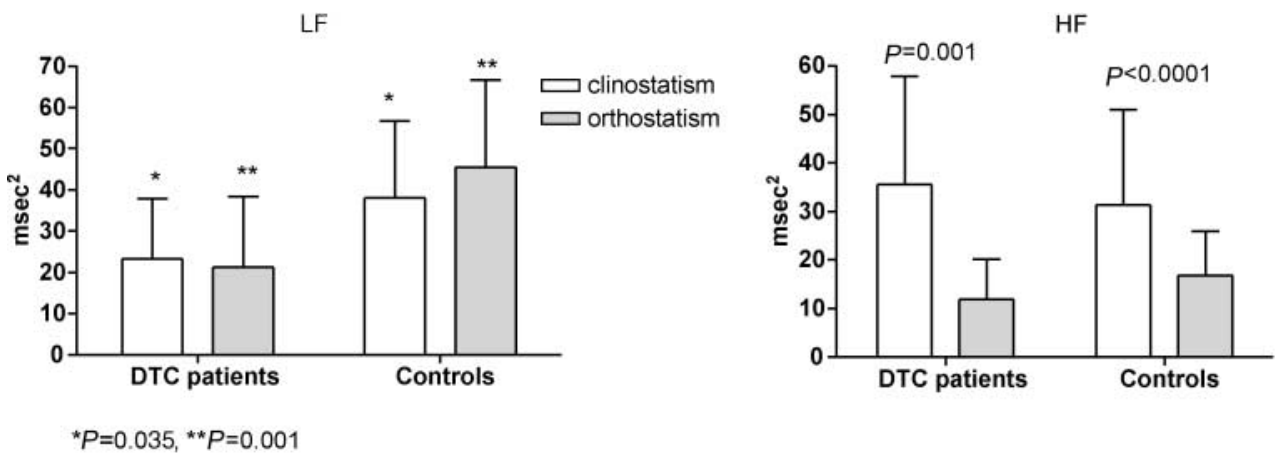

Figure 1 Low-frequency (LF) and high-frequency (HF) PSA components during clinostatism and orthostatism in 12 patients with DTC treated with suppressive doses of L-T4 and in 24 healthy controls. Statistical analysis: Mann-Whitney $U$ test for differences between patients and healthy controls ( $P$ values indicated by asterisks), and Wilcoxon test for the comparison between clinostatic and orthostatic data within groups ( $P$ values shown above the bars). 

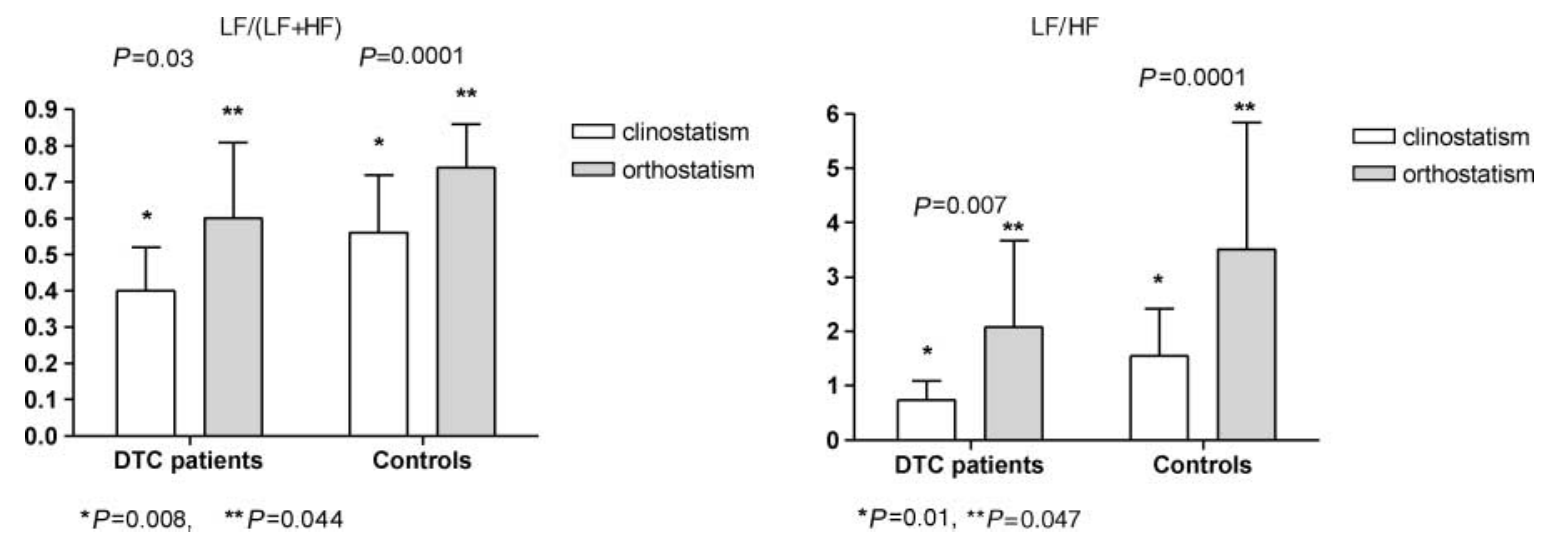

Figure $2 \mathrm{LF} /(\mathrm{LF}+\mathrm{HF})$ and LF/HF ratios in clinostatism and orthostatism in 12 patients with DTC treated with suppressive doses of L-T4 and in 24 healthy controls. For statistical analysis see legend to Fig. 1.

hormones is combined with greater left ventricular mass and enhanced inotropic response and cardiac output $(3,5,10)$. These changes in myocardial morphology and function, as well as slightly increased SBP, might determine a baroreflex-mediated impairment in sympathetic outflow and activity as observed in early experimental hyperthyroidism (36). Moreover, thyroid hormones may act directly on central nervous system areas that integrate autonomic function (33).

In conclusion, an impairment in sympatho-vagal balance, consisting of a sympathetic failure and altered autonomic mechanisms in response to the change from lying to standing, occurred in patients on longterm treatment with suppressive doses of $\mathrm{L}-\mathrm{T} 4$ for DTC. These changes possibly depend on a mild increase in SBP, which might modify sympathetic outflow acting on baroreceptors regulating HRV.

\section{Acknowledgements}

We are grateful to Professor Maria Vittoria Gianelli, Faculty of Medicine, University of Genova, for the linguistic revision of the manuscript.

\section{References}

1 Klein I. Thyroid hormone and the cardiovascular system. American Journal of Medicine $1990 \mathbf{8 8} 631-637$.

2 Polikar R, Burger AG, Scherrer U \& Nicod P. The thyroid and the heart. Circulation 199387 1435-1441.

3 Klein I \& Ojamaa K. Thyroid hormone and the cardiovascular system. New England Journal of Medicine 2001344 501-509.

4 Biondi B, Palmieri EA, Lombardi G \& Fazio S. Effects of thyroid hormones on cardiac function: the relative importance of rate, loading conditions, and myocardial contractility in the regulation of cardiac performance in human hyperthyroidism. Journal of Clinical Endocrinology and Metabolism 200287 968-974.

5 Fazio S, Palmieri EA, Lombardi G \& Biondi B. Effects of thyroid hormone on the cardiovascular system. Recent Progress in Hormone Research 200459 31-50.

6 Biondi B, Fazio S, Carella C, Sabatini D, Amato G, Cittadini A, Bellastella A, Lombardi G \& Sacca L. Control of adrenergic overactivity by beta-blockade improves the quality of life in patients receiving long term suppressive therapy with levo-thyroxine. Journal of Clinical Endocrinology and Metabolism 199478 1028-1033.

7 Ching GW, Franklyn JA, Stallard TJ, Daykin J, Sheppard MC \& Gammage MD. Cardiac hypertrophy as a result of long term thyroxine therapy and thyrotoxicosis. Heart 199675 363-368.

8 Sawin CT, Geller A, Wolf PA, Belanger AJ, Baker E, Bacharach P, Wilson PW, Nenjamin EJ \& D'Agostino RB. Low serum thyrotropin concentrations as a risk factor for atrial fibrillation in older persons. New England Journal of Medicine 1994331 1249-1252.

9 Biondi B, Palmieri EA, Fazio S, Cosco C, Nocera M, Sacca L, Filetti S, Lombardi G \& Perticone F. Endogenous subclinical hyperthyroidism affects quality of life and cardiac morphology and function in young and middle-aged patients. Journal of Clinical Endocrinology and Metabolism 200085 4701-4705.

10 Biondi B, Palmieri EA, Lombardi G \& Fazio S. Effects of subclinical thyroid dysfunction on the heart. Annals of Internal Medicine 2002 $137904-914$.

11 Sawin CT. Subclinical hyperthyroidism and atrial fibrillation. Thyroid 200212 501-503.

12 Ross DS. Subclinical thyrotossicosis. In Werner and Ingbar's The Thyroid: a Fundamental and Clinical Text, 7th edn, pp 1016-1020. Eds LE Bravermann \& RD Utiger. Philadelphia: Lippincott Williams \& Wilkins, 1996.

13 Biondi B, Fazio S, Carella C, Amato G, Cittadini A, Lupoli G, Sacca L, Bellastella A \& Lombardi G. Cardiac effects of long term thyrotropin-suppressive therapy with levothyroxine. Journal of Clinical Endocrinology and Metabolism 199377 334-338.

14 Fazio S, Biondi B, Carella C, Sabatini D, Cittadini A, Panza N, Lombardi G \& Sacca L. Diastolic dysfunction in patients on thy roid-stimulating hormone suppressive therapy with levothyroxine: beneficial effect of beta-blockade. Journal of Clinical Endocrinology and Metabolism $1995802222-2226$.

15 Shapiro LE, Sievert R, Ong L, Ocampo EL, Chance RA, Lee M, Nanna M, Ferrick K \& Surks MI. Minimal cardiac effects in asymptomatic athyreotic patients chronically treated with thyrotropin-suppressive doses of L-thyroxine. Journal of Clinical Endocrinology and Metabolism $1997822592-2595$.

16 Biondi B, Fazio S, Cuocolo A, Sabatini D, Nicolai E, Lombardi G, Salvatore M \& Sacca L. Impaired cardiac reserve and exercise capacity in patients receiving long-term thyrotropin suppressive therapy with levo-thyroxine. Journal of Clinical Endocrinology and Metabolism 199681 4224-4228.

17 Gullu S, Altuntas F, Dincer I, Erol C \& Kamel N. Effects of TSHsuppressive therapy on cardiac morphology and function: beneficial effects of the addition of $\beta$-blockade on diastolic dysfunction. European Journal of Endocrinology 2004150 655-661.

18 Mercuro G, Panzuto MG, Bina A, Leo M, Cabula R, Petrini L, Pigliaru F \& Mariotti S. Cardiac function, physical exercise capacity, 
and quality of life during long term thyrotropin-suppressive therapy with levo-thyroxine: effect of individual dose tailoring. Journal of Clinical Endocrinology and Metabolism 200085 159-164.

19 Task Force of The European Society of Cardiology and The North American Society of Pacing and Electrophysiology, Heart rate variability. Standards of measurement, physiological interpretation, and clinical use. European Heart Journal $1996 \mathbf{1 7}$ 354-381.

20 Stys A \& Stys T. Current clinical evidence of heart variability. Clinical Cardiology 199821 719-724.

21 Cacciatori V, Bellavere F, Pezzarossa A, Dellera A, Gemma ML, Thomaseth K, Castello R, Mughetti P \& Muggeo M. Power spectral analysis of heart rate in hyperthyroidism. Journal of Clinical Endocrinology and Metabolism $1996 \mathbf{8 1} 2828-2835$.

22 Inukai T, Takanashi K, Kobayashi H, Fujiwara Y, Aso Y \& Takemura Y. Power spectral analysis of variations in heart rate in patients with hyperthyroidism or hypothyroidism. Hormone and Metabolic Research $199830531-535$.

23 Burggraaf J, Tulen JHM, Lalezari S, Schoemaker RC, De Meyer PHEM, Meinders AE, Cohen AF \& Pijl H. Sympathovagal imbalance in hyperthyroidism. American Journal of Physiology 2001281 E190-E195.

24 Petretta M, Bonaduce D, Spinelli L, Vicario MLE, Nuzzo V, Marciano F, Camuso P, De Sanctis V \& Lupoli G. Cardiovascular haemodynamics and cardiac autonomic control in patients with subclinical and overt hyperthyroidism. European Journal of Endocrinology $2001145691-696$.

25 Goichot B, Brabdenberger G, Vinzio S, Perrin AE, Geny B, Schlienger JL \& Simon C. Sympathovagal response to orthostatism in overt and in subclinical hyperthyroidism. Journal of Endocrinological Investigation 200427 348-352.

26 Osman F, Franklyn JA, Daykin J, Chowdhary S, Holder RL, Sheppard MC \& Gammage MD. Heart rate variability and turbulence in hyperthyroidism before, during, and after treatment American Journal of Cardiology 200494 465-469.

27 Baselli G, Cerutti S, Civardi S, Lombardi F, Malliani A, Merri M, Pagani M \& Rizzo G. Heart rate variability signal processing: a quantitative approach as an aid to diagnosis in cardiovascular pathologies. International Journal of Biomedicine and Computers $19872051-70$.
28 Malliani A, Pagani M, Lombardi F \& Cerutti S. Cardiovascular neural regulation explored in the frequency domain. Circulation $199184482-491$.

29 Bernardi L, Leuzzi S, Radaelli A, Passino C, Johnston JA \& Sleight P. Low-frequency spontaneous fluctuations of R-R interval and blood pressure in conscious humans: a baroreceptor or central phenomenon? Clinical Sciences 199487 649-654.

30 Pagani M, Lombardi F, Guzzetti S, Rimoldi O, Furlan R, Pizzinelli P, Sandrone G, Malfatto G, Dell'Orto S, Piccaluga F, Turiel M, Baselli G, Cerutti S \& Malliani A. Power spectral analysis of heart rate and arterial pressure variability as a marker of sympatho-vagal interaction in man and in conscious dog. Circulation Research $198659178-193$.

31 Pomeranz B, Macaulay RJ, Caudill MA, Kutz I, Adam D, Gordon D, Kilborn KM, Barger AC, Shannon DC \& Cohen RJ. Assessment of autonomic function in humans by heart rate spectral analysis. American Journal of Physiology $1985 \mathbf{2 4 8}$ H151-H153.

32 Matsukawa T, Mano T, Gotoh E, Minamisawa K \& Ishii M. Altered muscle sympathetic nerve activity in hyperthyroidism and hypothyroidism. Journal of the Autonomic Nervous System 1993 23 207-211.

33 Kollai B \& Kollai M. Reduced cardiac vagal excitability in hyperthyroidism. Brain Research Bulletin 198820 785-790.

34 Maciel BC, Gallo L Jr, Marin Neto JA, Maciel LM, Alves ML, Paccola GM \& Iazigi N. The role of the autonomic nervous system in the resting tachycardia of human hyperthyroidism. Clinical Science $1987 \mathbf{7 2} 239-244$.

35 Lipsitz LA, Mietus J, Moody GB \& Goldberger AL. Spectral characteristics of heart rate variability before and during postural tilt. Circulation 199081 1803-1810.

36 Safa-Tisseront V, Ponchon P, Laude D \& Elghozi J-L. Contribution of the autonomic nervous system to blood pressure and heart rate variability changes in early experimental hyperthyroidism. European Journal of Pharmacology 1998352 247-255.

Received 23 November 2004

Accepted 25 February 2005 